\title{
Integración de la realidad virtual inmersiva en los Grados de Comunicación
}

\section{Integration of immersive virtual reality in Communication Degrees}

\author{
Dr. Ubaldo Cuesta Cambra \\ Director del Departamento de Comunicación Audiovisual y Publicidad II \\ (Universidad Complutense de Madrid) \\ Luis Mañas Viniegra \\ Profesor Asociado en el Departamento C.A.V.P. I \\ (Universidad Complutense de Madrid)
}

Fecha de recepción: 16 de marzo de 2016

Fecha de revisión: 10 de julio de 2016

Para citar este artículo: Cuesta Cambra, U. y Mañas Viniegra, L. (2016):

Integración de la realidad virtual inmersiva en los Grados de Comunicación, Icono 14, volumen 14 (2), pp. 1-21. doi: 10.7195/ri14.v14i2.953 


\section{ARTÍCULOS DE INVESTIGACIÓN}

\section{Resumen}

El Espacio Europeo de Educación Superior fomenta integrar las nuevas tecnologías en las metodologías docentes y mejorar la adquisición de competencias, cuestiones demandadas por los alumnos de la Universidad Complutense de Madrid, todos hoy con un perfil de nativo digital o "millennial". El artículo realiza un estudio sobre la implantación de la realidad virtual inmersiva en la parte práctica de las asignaturas de comunicación empresarial, específicamente en Comunicación de Crisis. La metodología se ha basado en encuestas y "focus group" a alumnos y profesores. Las conclusiones permiten afirmar que la realidad virtual inmersiva mejora las expectativas y el interés de los estudiantes y supone un incremento en las competencias adquiridas, permitiendo mejorar los niveles de empleabilidad, una de sus principales causas de insatisfacción. El uso consolidado de la telefonía móvil aconseja la elección de equipos de realidad virtual para estos dispositivos en vez de "cuevas" (C.A.V.E.) o equipos para videoconsolas.

Palabras clave: EEES - Educación en comunicación - Realidad virtual - Innovación didáctica - Aprendizaje interactivo - Comunicación de crisis - Industrias culturales - Videojuegos

\section{Abstract}

The European Higher Education Area promotes the integration of new technologies in didactic innovation and it aims to improve skills. It has been requested by students at the Complutense University of Madrid, who have a digital native profile or millennial. This article is a study about implementation of immersive virtual reality in the practical part of the subjects related to business communication. Specifically, it applied in the subject Crisis Communication. The methodology is a survey and three focus groups for professors and students. The conclusions say that the implementation of immersive virtual reality improves the expectations and interest of students. It also improves the skills acquired and the practical part of the subjects of communication improve employment of students of the Degree, which is one of their main causes of dissatisfaction. The full implementation of mobile telephony suggests using virtual reality devices adapted to them rather than "caves" (C.A.V.E.) or consoles.

Key Words: EHEA - Education in communication - Virtual reality - Didactic innovation - Interactive learning - Crisis communication - Cultural industries - Video games 


\section{Introducción y estado de la cuestión}

Desde la implantación del Espacio Europeo de Educación Superior (E.E.E.S.), la integración de las nuevas tecnologías en las universidades españolas ha sido una constante en un sistema orientado fundamentalmente a adquirir competencias e innovar educativamente, aunque lastrado en ocasiones por el salto generacional establecido entre profesores y alumnos, especialmente en lo referido a la gamificación o integración de juegos durante el aprendizaje para mejorar la estimulación, el esfuerzo y la participación del alumnado, a pesar de que "no todos los juegos están realmente gamificados, ni desde luego todas las aplicaciones que utilizan técnicas de gamificación son juegos como tal" (Cortizo, Carrero \& al., 2011:2).

Los estudiantes de los Grados en Comunicación, todos hoy nativos digitales o "millennials", consideran como causas principales de su insatisfacción la adopción de metodologías docentes excesivamente teóricas, no vinculadas con el uso de la tecnología, en ocasiones como consecuencia de una infraestructura insuficiente, y unos índices de empleabilidad mejorables. De este modo, el Consejo Social de la Universidad Complutense de Madrid (U.C.M., 2014) señala que los alumnos de las carreras de Periodismo, Comunicación Audiovisual y Publicidad y Relaciones Públicas son los que muestran menor satisfacción ante la relación entre empleo y formación de todas las áreas de ciencias sociales de la Universidad tras Educación Social.

La plena integración de esas tecnologías en los programas académicos dirigidos a estudiantes con perfil "millenial" no puede concebirse hoy sin la utilización de los videojuegos, un sector que desde el año 2009 se incluye oficialmente en España dentro de la categoría de Industrias Culturales y Creativas, que supusieron un 3,5\% del P.I.B. en 2012, según los últimos datos publicados por el Ministerio de Educación, Cultura y Deporte (M.E.C.D., 2014). Su reconocimiento cultural implicó que comenzara a ligarse al aprendizaje, la educación y la cultura más allá del mero entretenimiento. El M.E.C.D. (2015) cuantifica en un 43,5\% su uso entre los jóvenes de entre 15 y 24 años y en un 45,6\% entre los estudiantes que juegan, al menos, una vez al mes, siendo el colectivo joven estudiantil el segmento de población en el que alcanza mayor penetración sobre el total. E.A.E. (2015) señala que el sector facturó en 2014 en España 763 millones de Euros, procediendo el 94,1\% 
de las ventas de juegos para videoconsolas, siendo Madrid la comunidad autónoma en la que se produce mayor gasto por habitante.

Durante la última década, los videojuegos han demostrado su utilidad en el aprendizaje de idiomas, desarrollo de la creatividad o de la agilidad mental. Sin embargo, su aplicación en una asignatura de Grado, específicamente Comunicación de Crisis, que es la que aquí se plantea, requiere una evolución de los videojuegos hacia la simulación basada en la realidad virtual inmersiva, aunque sin descartar la atracción que para los jóvenes suponen los videojuegos.

La elección de la asignatura Comunicación de Crisis no es arbitraria, puesto que es optativa de último curso del Grado en Publicidad y Relaciones Públicas de la U.C.M., disponiendo los estudiantes de los conocimientos y competencias necesarios para afrontar una asignatura que requiere una combinación de conocimientos teóricos, habilidades de interacción y aplicación práctica en la toma de decisiones. A.N.E.C.A. (2005: 204, 328, 335) ya señalaba la importancia de la gestión de la comunicación en agencias, organizaciones o consultoras en cuanto a la alta empleabilidad y buena remuneración, tendencia que ha continuado al alza desde entonces. Además, señalaba como la competencia profesional más valorada la "capacidad y habilidad para utilizar las tecnologías y técnicas comunicativas" y como una de las "exigencias de formación" de los entonces nuevos títulos de Grado "el acercamiento a la realidad profesional mediante la experimentación a través de prácticas [...] en laboratorios docentes". Hay que considerar que los "estudiantes son capaces de dominar, retener y generalizar los nuevos conocimientos de una manera mucho más efectiva si se ven envueltos activamente en la experiencia de aprendizaje" (Otero \& Flores, 2011:194).

Si consideramos que la realidad virtual se puede utilizar en una simulación (Pantelidis, 1996) para evitar los errores significativos que podría acarrear la enseñanza en un entorno real y que la interacción con el modelo representado es más motivante y permite crear experiencias participativas, como en el caso que nos ocupa, su aplicación sería completamente válida.

A pesar de que algunos autores manifiestan su disconformidad con el término realidad virtual por considerarlo ambiguo (Castañares, 2007) y vinculado, en 
ocasiones, con lo irreal por implicar una oposición a lo actual y a la solución de un problema (Lévy, 1999), lo cierto es que desde los años 80 del siglo pasado se comienza a utilizar el término para relacionarlo con una tecnología que permitía la percepción de un entorno diferente al físico (Lanier, 1988), pero en el que se utilizan, de igual modo, los cinco sentidos. Por ello, diversos autores (Krueger, 1983; Walker, 1988) se decantaban por el término "realidad artificial", a pesar de que no llegó a ser el mayoritariamente utilizado.

En cualquier caso, existe un amplio consenso al afirmar que lo virtual intenta representar diversas situaciones que se producen en la realidad con la mayor similitud posible, de ahí su aplicación práctica a la formación de futuros profesionales de la comunicación empresarial. Este enfoque ha permitido la evolución del concepto hasta vincularse con los denominados sistemas de interacción entre usuarios o humanos y ordenadores o tecnología (I.H.O. o H.C.I., en su acrónimo inglés), que permiten "determinar cómo podemos hacer la tecnología más usable para la gente" (Cleveland,1993). De nada valdría la creación de una realidad virtual que represente fidedignamente las situaciones que los profesionales tendrán que afrontar si no existe una facilidad de uso, ésta no es intuitiva o los elementos tecnológicos auxiliares dificultan la movilidad.

El grado de realismo gráfico a la hora de representar la realidad ha sido uno de los puntos débiles de la realidad virtual aplicada a la formación, disminuyendo la implicación de los estudiantes al reducir considerablemente el atractivo del contenido de la representación, como han demostrado en una relación inversamente proporcional los videojuegos. Resulta fundamental otorgar gran importancia al realismo y la viveza de las imágenes generadas (Sheridan,1992, 1996; Steuer, 1992; Biocca, Kim \& Levy, 1995; Slater \& Usoh, 1993).

En esta línea, Castañares (2011:62), establece una definición de "realidad virtual" aplicable a la finalidad aquí perseguida al asociarla a las representaciones digitales "que pretenden producir el mismo tipo de efectos perceptivos que los objetos sensibles de la realidad física de la vida cotidiana y que reacciona ante la acción del hombre de manera semejante a como lo hace esa realidad". 
Para que puedan producirse este tipo de efectos, es necesario asegurar, más allá de la mera interactividad, su carácter inmersivo o sensación que experimenta el usuario de encontrarse dentro de la situación de realidad virtual, que es precisamente una de las tres características asignadas a la mencionada realidad virtual (Heim, 1993). En efecto, esa ambigüedad asociada al término comentada anteriormente implica que no todos los dispositivos e infraestructuras utilizados al hablar de realidad virtual cumplen con ese requisito inmersivo. Durante la última década se han utilizado mayoritariamente las "Cave Automatic Virtual Environment" (C.A.V.E.), que combinan el uso de gafas estereoscópicas con la proyección de imágenes en las paredes de un cubo, en cuyo interior se sitúa el usuario, garantizando la tridimensionalidad del espacio y de los objetos como paso previo a la inmersión, algo que los hace más costosos que otras soluciones virtuales. No podemos obviar que las C.A.V.E. reducen su utilización a la propia infraestructura física y a un número limitado de usuarios que pueden interactuar simultáneamente. Al contemplar su elevado coste de adquisición, resultan de gran interés para los investigadores, pero son difícilmente transferibles a la docencia en las aulas.

Dentro de esa inmersión, estos simuladores de realidad virtual permiten al sujeto libertad de movimiento, algo que un mero software o los denominados simuladores empresariales basados en la interfaz de un ordenador, que también permiten voluntad en la acción del usuario, no consiguen, a pesar de que es la solución hasta la fecha utilizada mayoritariamente en todos los estudios de postgrado tipo Máster relacionados con las disciplinas de la gestión empresarial, el marketing o la comunicación.

Tampoco podemos olvidar que la realidad virtual no está exenta de riesgos para las personas que adquieren una formación a través de ella, más allá de "la distopía, que no es otra cosa que la resistencia y el miedo a lo desconocido" (Cuadrado, 2011:8). En referencia a la tercera cualidad asignada a la realidad virtual (Heim, 1993), su carácter intensivo, algunos estudios (Havron \& Butler, 1957; Ungs, 1987; Kenedy, Lane, Lilienthal, Berbaum \& Hettinger, 1992; Regan, 1995; Repperger, Gilkey, Green, La Fleur \& Haas, 2003), relacionados mayoritariamente con el entrenamiento de pilotos mediante simuladores de vuelo basados en la realidad virtual, señalan efectos secundarios como náuseas, mareos, dolores de cabeza o alteracio- 
nes en la vista y el estómago durante varias horas tras la simulación. Esos inconvenientes, aunque soportables para la mayoría de los sujetos, se ven reducidos al repetir su participación (Regan \& Price, 1993).

En esa línea, la de la disminución de los efectos secundarios a medida que se incrementa la exposición a su uso, se pronuncian numerosos autores (Sharples, Cobb, Moody \& Wilson, 2008; Howart \& Hodder, 2008; Dong, Yoshida \& Stoffregen, 2011) al aplicar estos efectos a los videojuegos con cascos inmersivos. En los experimentos realizados por Guerrero \& Valero (2013:170-173) tan sólo obtuvieron resultados preocupantes en cuanto a las molestias generadas por la realidad inmersiva en un videojuego en el grupo que se encontró expuesto durante 5 horas seguidas, con un $30 \%$ de casos con pérdida de equilibrio, ansiedad ( $20 \%$ de los casos) e, incluso, abandono (30\% de los casos) y, en menor medida, en los que se exponían por primera vez a la realidad virtual inmersiva.

La evolución de los equipos en los últimos años ha posibilitado que la realidad virtual sea cada vez menos molesta para el usuario a la hora de realizar esa inmersión, desde el "ultimate display" (Sutherland,1968), el primer casco de realidad virtual, pasando por el "videoplace" (Krueger, 1985), el guante de datos "data glove" creado por Sandin en 1977 (Sturman \& Zeltzer, 1994), los sensores de posición "polhemos" hasta el nacimiento de las C.A.V.E. en la Universidad de Illinois en 1992 y la conectividad a Internet de la realidad virtual, que se produjo con la creación del lenguaje de programación "virtual reality modeling language" o V.R.M.L. (Bell, Parisi \& Pesce, 1992).

Los hermanos Parés (2010) afirman que "aquello que genera el impulso por desarrollar una cierta tecnología son las ideas que hay detrás" $\mathrm{y}$, en efecto, en el área de conocimiento de Comunicación Audiovisual y Publicidad son esas ideas las que permiten que la tecnología pueda ser aplicada. Se favorece así el aprendizaje, incentivando la implicación del alumnado mientras, simultáneamente, obtiene una mayor preparación para resolver situaciones reales que tendrá que afrontar en su futuro profesional, recreando todas las características de la situación que afectarán a los cinco sentidos y siendo las habilidades necesarias para su utilización en el ámbito educativo un 50\% visuales, 20\% auditivas y 30\% kinestésicas (González \& 


\section{ARTÍCULOS DE INVESTIGACIÓN}

Chávez, 2011:132). Es un proceso de aprendizaje calificado como "conocimiento en primera persona" (De-Antonio, Villalobos \& Luna, 2000:2).

Cualquier simulación debe cumplir los criterios de un modelo con similitud con el fenómeno estudiado (Whicker \& Sigelman, 1991), en nuestro caso un modelo físico, y analítico (Poole \& Szymankiewicz, 1977), así como la simulación de sistemas 0 representación de la estructura simulada y uso de un ordenador por parte de una 0 varias personas. La metodología de actuación ensayo-error que posibilita un simulador evita el riesgo que asumiría una organización al experimentar con el sistema real en un entorno natural. En la inmensa mayoría de las ocasiones, se utiliza la simulación por ordenador (Whicker \& Sigelman, 1991; McHaney, 1991; Ripley, 1987; Matloff, 1988; Poole \& Szymankiewicz, 1977; Shannon, 1975) y, en concreto, una simulación persona-ordenador, que ubica a la persona en un contexto específico al que debe ofrecer respuesta y reaccionar a las situaciones hasta que se produzca el aprendizaje (Wicker \& Sigelman, 1991; Earnshaw, Gigante \& Jones, 1993; Kalawsky, 1993; Vince, 1995), debido a los altos costes que supone hacerlo persona-persona.

En efecto, sólo podemos asegurar que el modelo sea lo más similar posible al fenómeno a partir del estudio de casos prácticos específicos que se han repetido a lo largo del tiempo, que nos proporcionan una experiencia a la hora de determinar cuáles son los aciertos y errores, junto a los modelos de relaciones públicas a nivel académico e investigador que se encuentran plenamente consolidados y que son aplicables a otros casos similares que pudieran producirse en el futuro. De este modo, el entorno inmersivo será beneficioso para los alumnos al permitir la construcción de "un ambiente de aprendizaje que los anima a la construcción conjunta de conocimiento, apoyándose en la reflexión crítica y la interacción social con otros estudiantes" (Jerónimo, Andrade \& Robles, 2011:27).

\section{Material y métodos}

La investigación analiza la expectativa que genera en el alumnado de la Universidad Complutense de Madrid la posible introducción de la realidad virtual inmersiva en la asignatura de Comunicación de Crisis como experiencia piloto dentro del Grado en Publicidad y Relaciones Públicas en el contexto del E.E.E.S. 
Integración de la realidad virtual inmersiva en los Grados de Comunicación | 9 ARTíCULOS DE INVESTIGACIÓN

En cuanto a los objetivos específicos, se plantean los siguientes:

- Conocer los principales motivos de insatisfacción de los estudiantes de la U.C.M. en relación con la Titulación.

- Determinar la expectativa de mejora docente que supondría la introducción de la realidad virtual inmersiva.

- Analizar la idoneidad de la asignatura de Comunicación de Crisis para la implantación de la realidad virtual inmersiva.

- Seleccionar la infraestructura y equipamiento adecuados en el contexto actual de las universidades españolas y, en concreto, de la U.C.M.

- Realizar una evaluación de los riesgos asociados al uso de la modalidad de realidad virtual elegida.

La metodología aplicada tras el análisis bibliográfico relacionado con el estado de la cuestión incluye una combinación de técnicas cuantitativas y cualitativas en tres fases consecutivas durante el curso académico 2015/2016.

La primera fase consiste en un análisis de contenido de información secundaria en lo referente a estadísticas generadas por la propia Universidad relativas a la satisfacción de alumnos a través del programa Docentia.

La segunda fase se centra en la encuesta realizada a los alumnos para generar información primaria relacionada con los hábitos de uso de los videojuegos y las posibilidades de éxito de implantación de la realidad virtual inmersiva en la asignatura de Comunicación de Crisis. A pesar de que son 1.029 los alumnos matriculados en el Grado en Publicidad y Relaciones Públicas de la U.C.M. durante el curso académico 15/16, el universo objeto de estudio se encuentra formado por los 423 alumnos que se encuentran en 3er. y $4^{\circ}$ curso, aplicando el filtro de que hayan cursado algunas de las asignaturas relacionadas con la comunicación empresarial durante el 1er. cuatrimestre para poder tener una opinión formada sobre la docencia 
que han recibido en el marco de la Titulación y la Universidad, específicamente. De igual modo, se han eliminado las duplicidades producidas por alumnos que cursan dos asignaturas simultáneamente, debido al impacto que las asignaturas optativas tienen en este sentido.

Se ha utilizado el formato de encuesta autoadministrada y seleccionada a través de su difusión mediante la lista de distribución de correo electrónico del campus virtual, que ha sido el filtro empleado. La encuesta estuvo activa en la Red durante el mes de enero de 2016, una vez finalizada la docencia del primer cuatrimestre. El tamaño final de la muestra viene marcado por los 206 cuestionarios válidos completados, que representan un margen de error del 4,9\% para un $95 \%$ de N.C., y que fueron sometidos a un proceso de revisión y validación. Se ha limitado la cumplimentación a una única encuesta por dirección IP y se han eliminado aquéllas con una presencia no justificada de preguntas no contestadas o incongruencias en sus respuestas.

El cuestionario se ha elaborado utilizando el "plugin" FSQM Pro y un lenguaje de programación PHP. Se elaboró con 15 preguntas de diversa índole, cerradas, abiertas y de valoración con escala Likert con cinco opciones, junto a las correspondientes preguntas filtro y de control. Las preguntas se encontraban estructuradas a partir de tres bloques temáticos: uso de videoconsolas y videojuegos, uso de realidad virtual y evaluación de la docencia y de sus posibilidades de mejora.

La tercera fase es la realización de "focus group" como técnica cualitativa no estructurada y directa para obtener información a partir de la interacción de los componentes de tres grupos de 8 personas cada uno, estableciendo una cuota de paridad entre mujeres y hombres formados, por un lado, por el mismo perfil de los alumnos encuestados $\mathrm{y}$, por otro, por profesores con docencia en alguna de las asignaturas relacionadas con la comunicación empresarial, con el objetivo de extraer conclusiones sobre las dificultades y beneficios que los alumnos (dos grupos) y el P.D.I. aprecian en relación con la implantación de la experiencia piloto. 


\section{Resultados}

El Grado en Publicidad y Relaciones Públicas de la U.C.M. debe, en el marco del E.E.E.S., continuar con una necesaria y progresiva implantación de nuevas tecnologías en las metodologías y contenidos docentes de sus planes de estudio, contemplando las dificultades presupuestarias de estos años de crisis económica, pero sin olvidar que la captación del talento requiere ofrecer una respuesta a las expectativas del alumnado. En este sentido, la adquisición de competencias que figura en sus planteamientos exige que los alumnos se encuentren plenamente capacitados para aplicar profesionalmente sus conocimientos al finalizar sus estudios de Grado, hecho que permitirá, en última instancia, la mejora de los índices de empleabilidad de la Titulación.

La revisión de las encuestas realizadas a los alumnos del Grado en Publicidad y Relaciones Públicas por la U.C.M. en el marco del programa "Docentia" revela que algunos de los aspectos a mejorar son la "formación recibida en relación con las competencias vinculadas a la Titulación", con una puntuación media de 5,37 sobre 10,00 en el curso académico 2013/2014, las "instalaciones y recursos de apoyo a la docencia", con una puntación de 4,38, la "metodología docente del profesorado", con una puntuación media de 5,42 o las "prácticas externas o preprofesionales", con 4,77 . Todos estos aspectos guardan una relación directa y podrían mejorar con la implantación de la realidad virtual inmersiva en la docencia.

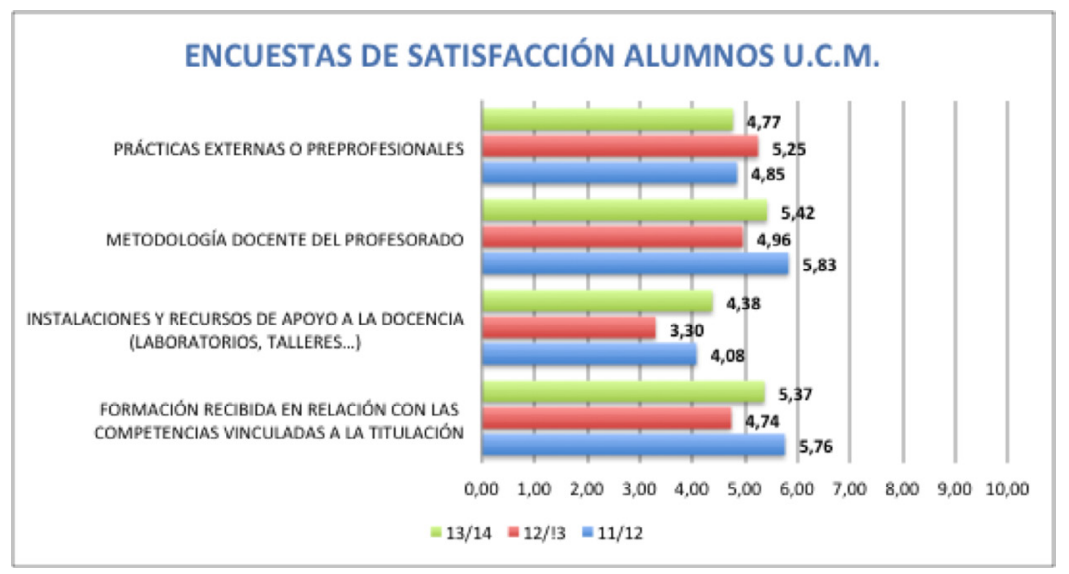

Gráfico 1: Satisfacción de los estudiantes del Grado en Publicidad y RR.PP. en la U.C.M. Elaboración propia a partir de U.C.M. (2015). 
Los datos extraídos de la encuesta realizada permiten identificar cuál sería el dispositivo más idóneo para la implantación de esa realidad virtual inmersiva. A pesar de que el 55,56\% de los encuestados manifestaron disponer de videoconsola en su domicilio durante el curso, mayoritariamente PlayStation, la tipología de estudiantes de la U.C.M., con un elevado porcentaje de alumnos procedentes de otras comunidades autónomas, hace que sean muchos los que disponen de la misma en sus hogares familiares de origen, por lo que la mitad de los alumnos estaría sujeto a su utilización exclusiva en el aula. Por otro lado, de los alumnos que manifestaron no disponer de videoconsola en su domicilio durante el curso, el 54,39\% sí dispone de ordenador, dispositivo que no permitiría emprender una iniciativa como la aquí perseguida. En última instancia, hay que considerar que el $100 \%$ de los alumnos dispone de teléfono móvil, todos con formato "smartphone".

En cuanto a los hábitos de uso, el 59,22\% de los encuestados emplea un máximo de 1 hora semanal en jugar a su videoconsola $y$, mayoritariamente, la han empleado durante el año 2015 en juegos de estrategia y deportes, señalando una amplia variedad de títulos dentro de estas dos categonías. Un 74,76\% afirma haber utilizado alguna vez videojuegos en los que existe algún componente de realidad virtual, aunque sin ser ésta completamente inmersiva, como “Just Dance", "Guitar Hero", "Sing Star" o “Wii Sports".

Una vez explicados los diversos equipos que se podrían utilizar para esta experiencia piloto, el 32,04\% consideró que su uso le resultaría fácil o extremadamente fácil y una gran mayoría, el 51,46\% lo considero ni fácil ni difícil, por lo que, en este sentido, no existen grandes dificultades que no puedan solventarse con un aprendizaje previo.

Otra de las cuestiones que afectan a la implantación de esta investigación es si habitualmente los estudiantes de la U.C.M. juegan conectados con otras personas que se encuentran a distancia o que, sencillamente, no conocen, tendencia cada vez mayor en los perfiles más jóvenes de usuarios de videoconsolas. Mayoritariamente, el $64,08 \%$ afirma no jugar nunca conectado en red, lo que limita la interconexión de estudiantes de cualquier universidad española o internacional en la utilización del simulador, sin limites geográficos ni de horarios. Parece que este aspecto habrá que afrontarlo en una segunda fase de la investigación, cuando los mas jóvenes habituados a jugar en red hayan comenzado sus estudios en la Universidad. 
Es necesario dilucidar si la aplicación de la realidad virtual a las asignaturas relacionadas con la comunicación empresarial supondría una mejora de competencias, un incremento en el interés del alumnado y una mayor satisfacción en relación con los estudios cursados. En la línea de la estadística mostrada inicialmente en las encuestas de satisfacción del programa "Docentia", los encuestados valoran que el Grado cursado a nivel global es teórico o excesivamente teórico en un $60,20 \%$ de los casos, mientras que el $39,81 \%$ considera que ha recibido una formación teórico-práctica. Al analizar la asignatura más genérica relacionada con la comunicación empresarial, la de Comunicación Corporativa, sólo el 20,39\% del total la considera excesivamente teórica o teórica.

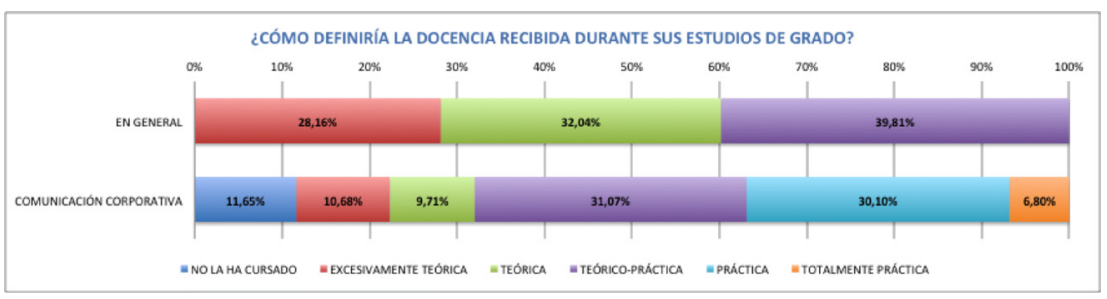

Gráfico 2: Percepción de la docencia recibida. Elaboración propia.

Si atendemos a las asignaturas más especializadas dentro de la comunicación empresarial $\mathrm{y}$, específicamente, a las respuestas de quienes ya las han cursado, la percepción actual del alumnado ya refleja ese componente más práctico o totalmente práctico en un 8,16\% en Comunicación Institucional e Imagen Pública, pero especialmente en las dos asignaturas optativas: Formación de Portavoces $(30,00 \%)$ y Comunicación de Crisis $(26,00 \%)$, siendo esta última donde se produce el mayor número de respuestas que la califican como "totalmente práctica", con un 12,50\% de las respuestas de quienes la han cursado.

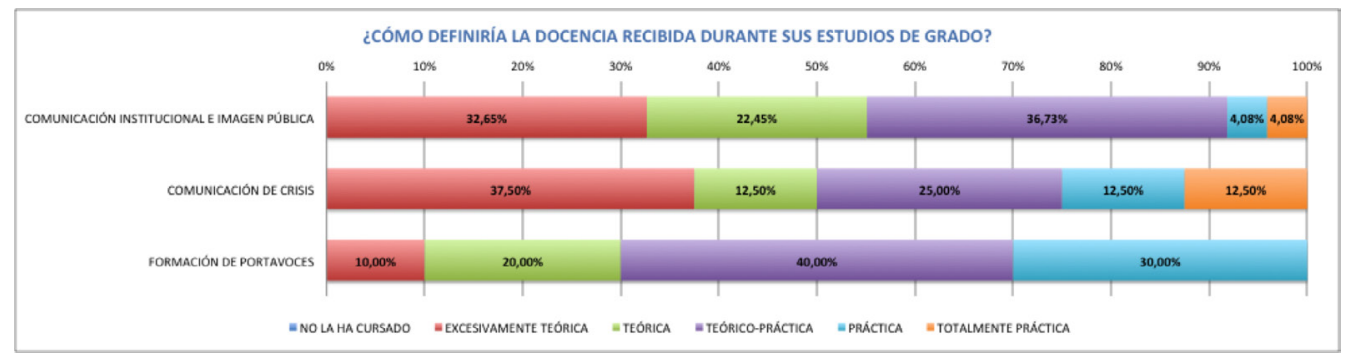

Gráfico 3: Percepción de la docencia recibida. Elaboración propia. 
Llegados a este punto, es necesario saber si la adquisición de competencias hubiera mejorado con la práctica de casos reales, sin mencionar en este caso la realidad virtual. El 73,79\% afirma con rotundidad que sí y tan sólo el 8,74\% considera que no. Las competencias adquiridas, además, presentan una mayor solidez y permanencia cuando la implicación del alumnado es mayor. En este sentido, un $77,67 \%$ de los encuestados afirmó encontrarse de acuerdo o totalmente de acuerdo con el hecho de que su interés en las asignaturas relacionadas con la comunicación empresarial sería mayor si en esa parte práctica de la asignatura, además de enfrentarse a casos reales, utilizasen la realidad virtual inmersiva.

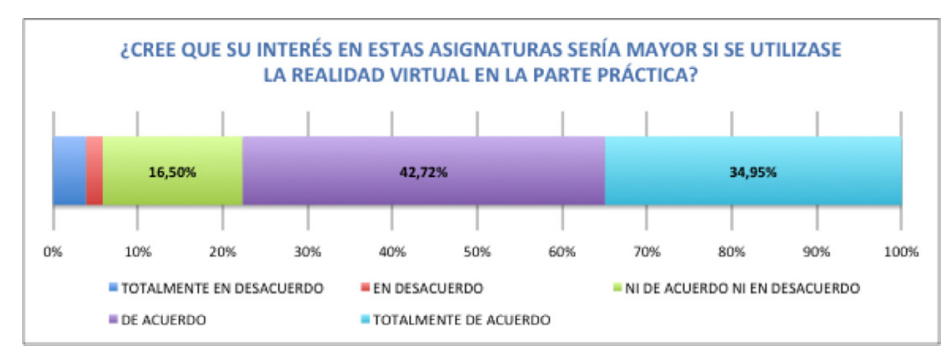

Gráfico 4: Interés en cursar asignaturas basadas en la realidad virtual inmersiva. Elaboración propia.

El análisis de la satisfacción experimentada por los estudiantes que ya han cursado la asignatura de Comunicación de Crisis refleja que el 45,55\% de los alumnos la valoran con una nota de notable ( 7 y 8 ) y el $18,89 \%$ de sobresaliente (9 y 10). Al considerar la aplicación de la realidad virtual, la nota sobresaliente se ampliaría hasta el 50,00\%. Si atendemos a los alumnos más insatisfechos, el 15,56\% que suspendieron (1-4) la docencia recibida, al aplicar el programa de realidad virtual se verían reducidos a menos de la mitad, tan sólo un 7,15\%.

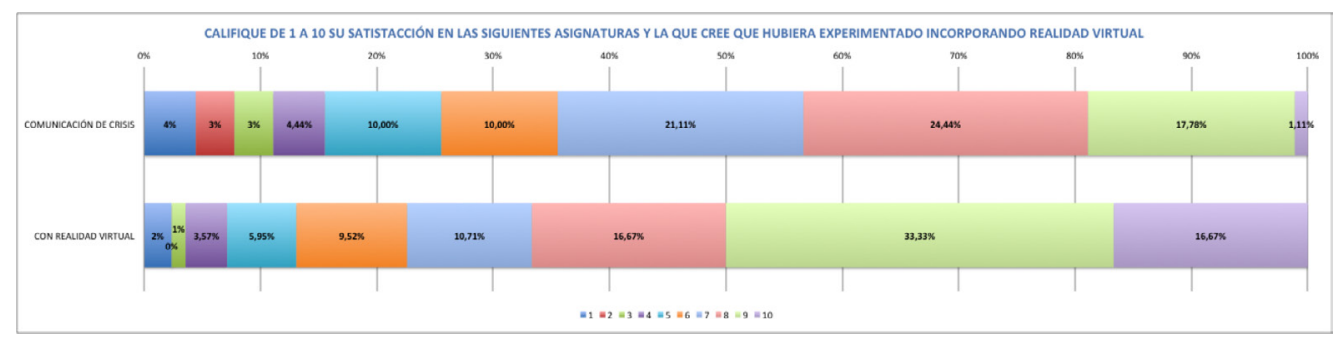

Gráfico 5: Satisfacción con la docencia recibida y esperada. Elaboración propia. 
Integración de la realidad virtual inmersiva en los Grados de Comunicación | 15

ARTÍCULOS DE INVESTIGACIÓN

Las inquietudes y posibles inconvenientes a la hora de implantar esta investigación se analizaron en los dos "focus group" formados por los alumnos. Se extrajeron como principales conclusiones a partir de las afirmaciones expresadas por la mayoría del grupo las relacionadas con las ventajas para su aprendizaje al practicar situaciones reales ("Se aprende mejor, aprendes a enfrentarte de alguna manera con las situaciones de la vida real."), además de poder realizar las prácticas sin estar sujetos a la saturación de las aulas ("Al ser tantos, era difícil que todos pudiéramos hacer muchas cosas. En cambio, con la realidad virtual podemos practicar todos."), la mejora que experimentaría su interés por las clases ("0jalá la realidad se llevase a las aulas para hacer más atractivas las asignaturas y más lúdico el aprendizaje."), la necesidad de incorporar más tecnología ("Falta, desde luego, incorporar nuevos modelos tecnológicos de aprendizaje.") en una metodología docente que reconocen que ya incorpora un combinación de teoría y práctica, aunque la perciben como escasa ("Esta deficiencia práctica constituye el principal problema de nuestro Grado.") o la disminución en la dedicación y la preferencia de jugar online a medida que el jugador va cumpliendo años ("Entre los 15 y los 17 años jugábamos a la consola al menos dos horas al día y siempre online. Ahora, sólo un par de horas a la semana con los amigos en casa.").

El "focus group" realizado entre profesores de asignaturas afines a la comunicación empresarial transcurrió por los caminos del coste de su implantación y de la dificultad en su desarrollo, de modo que implique una simulación creíble de situaciones que el alumnado deberá resolver posteriormente en un entorno real, así como los posibles efectos secundarios que pudieran producirse, junto a la preocupación por la necesidad de actualizar sus competencias tecnológicas para su implementación en planes de estudios ya instaurados.

\section{Discusión y conclusiones}

Los alumnos del Grado en Publicidad y Relaciones Públicas de la U.C.M. muestran una clara aspiración de mejora en cuanto a la adquisición de competencias orientadas hacia la práctica profesional para poder afrontar en el futuro su incorporación al mercado laboral e incrementar su empleabilidad, fomentando el uso de las nuevas tecnologías y otros recursos de apoyo a la docencia. De este modo, 


\section{ARTÍCULOS DE INVESTIGACIÓN}

consideran que su interés y satisfacción mejoraría sustancialmente si, además, contaran con la aplicación de un simulador de realidad virtual inmersiva en las asignaturas relacionadas con la comunicación empresarial, específicamente en Comunicación de Crisis, que aún no ha sido desarrollado por ninguna organización y que deberá constituirse en breve como un proyecto de investigación. La mejora en los índices de empleabilidad que este programa experimental tendría es consecuencia directa de los múltiples errores que las corporaciones de diversa índole cometen cada año en la comunicación ejercida sobre las situaciones de crisis a las que deben enfrentarse y que ponen de manifiesto la necesidad de contar con profesionales plenamente formados en esta materia.

Para la implantación de esta experiencia piloto, es necesario dilucidar cuál es el equipo de realidad virtual más indicado. La comodidad de uso de los equipos y el avance en la velocidad de procesamiento han sido notables en la última década y durante los años 2015 y 2016 se van a comercializar las tres alternativas más polivalentes para alcanzar los objetivos propuestos de simulación a partir de realidad virtual inmersiva: una C.A.V.E. de realidad virtual, un visor para utilizar conjuntamente con la videoconsola y los dispositivos desarrollados para el uso del teléfono móvil.

Las dificultades presupuestarias en las que hoy se enmarcan las universidades españolas hacen inviable la opción de la C.A.V.E., que además presenta el inconveniente de limitar su utilización al aula y a un número reducido de alumnos. Durante años, muchas empresas públicas y organismos de carácter tecnológico se lanzaron a la construcción y equipamiento de C.A.V.E. de realidad virtual al amparo de fondos europeos, mientras las universidades siguieron el mismo camino con su incorporación a un desarrollo tecnológico que auguraba grandes avances campos como las ingenierías, la arquitectura, la medicina o la psicología, en tanto que permitía realizar un entrenamiento de cara a la preparación de situaciones reales que posteriormente se producirían en el desempeño de su labor profesional. Sin embargo, son muchos los organismos que tras la construcción de las "cuevas" esperaban que las empresas las alquilasen para la formación continua de sus empleados y que las universidades y escuelas de negocio hiciesen lo propio para la formación de sus estudiantes, algo que no se produjo por la falta de aplicaciones específicas que complementasen esa infraestructura tecnológica. 
La segunda opción parecía inicialmente la más idónea a partir de la calidad gráfica del visor "Morpheus" de Sony para su videoconsola PlayStation. Sin embargo, sólo la mitad de los alumnos disponen de videoconsola en su domicilio durante el curso académico, por lo que el simulador estaría sólo disponible en el aula, además de tener un coste de desarrollo superior al de la tercera opción.

En el año 2015 se lanzaba en Estados Unidos por primera vez un dispositivo de realidad virtual inmersiva cuyo soporte era el teléfono móvil, que el $100 \%$ de los alumnos poseen, lo que reduce considerablemente el coste de la inversión al dispositivo que sirve de soporte, facilita su adquisición y préstamo al alumnado, toda vez que facilita el desarrollo del "software" del simulador, más ágil, más flexible y más barato que las dos opciones anteriores. Tanto el "Gear VR" de Samsung como el "Cardboard" de Google facilitan junto a la telefonía móvil la utilización dentro y fuera del aula, en cualquier lugar, con la conectividad necesaria para establecer prácticas en red, en cualquier lugar y en cualquier momento. Además, Google ha lanzado en Estados Unidos, Canadá, Suecia, Hawaii y Alasca a mediados del año 2015 su programa "Expeditions", consistente en la difusión del dispositivo "Cardboard" en los centros educativos, cuya implicación abre una vía de colaboración con las universidades europeas para continuar este camino de desarrollo y difusión emprendido y puede ser viable su implicación en este proyecto.

A pesar de los inconvenientes físicos que un dispositivo de realidad virtual inmersiva puede generar, hay que considerar que los equipos domésticos que permiten simular una visión en tres dimensiones se han popularizado, lo que ha generado un hábito de uso en las generaciones más jóvenes suficiente para reducirlos al mínimo, si es que se produjeran. Hay que considerar con respecto a los estudios revisados que los simuladores aéreos suponen la simulación de un desplazamiento mientras el individuo permanece inmóvil, pero en la aplicación aquí defendida, el individuo es quien decide los movimientos de su cuerpo a la vez que los realiza con su aparato psicomotriz, lo que va mucho más allá del mero control de la acción al que aludían Dong, Yoshida y Stoffregen (2011) como elemento reductor de la sensación de malestar experimentada. 


\section{ARTÍCULOS DE INVESTIGACIÓN}

En cualquier caso, será necesario descartar inicialmente, por precaución, a personas con problemas de salud previos relacionados con estos síntomas y se limitará la duración de cada sesión práctica de simulación a una hora y media para evitar los efectos de cansancio y vista cansada. Aunque en una situación de comunicación de crisis el cansancio afecta a la lucidez en la toma de decisiones, no parece ético exponer artificialmente a los participantes en una fase de formación a este tipo de inconvenientes a los que tienen que enfrentarse profesionales con una experiencia contrastada.

\section{Referencias}

A.N.E.C.A. (2005). Libro Blanco de Títulos de Grado en Comunicación. Madrid: ANECA. Recuperado de http://goo.gl/f4QRio

Bell, G., Parisi, A. \& Pesce, M. (1995). The virtual reality modeling lenguaje versión 1.0 specification. Recuperado de http://goo.gl/swRdSM

Biocca, F. \& Levy, M.R. (1995). Virtual Reality as a Communication System. En Biocca, F. \& Levy, M.R. (Eds.), Communication in the Age of Virtual Reality. Hillsdale (15-31). NJ: Lawrence Erlbaum Associates, Inc.

Castañares, W. (2007). Cultura visual y crisis de la experiencia. CIC Cuadernos de Información y Comunicación, 12, 29-48.

Castañares, W. (2011). Realidad virtual, mímesis y simulación. CIC Cuadernos de Información y Comunicación, 16, 59-81. doi: 10.5209/rev_CIYC.2011.v16.3

Cleveland, D. \& Cleveland, N. (1993). A Headtracking Device for the Eyegaze Eyetracking System. En Proceedings of the Fifth International Conference on Human-Computer Interaction - Poster Sessions: Abridged Proceedings (p. 239). Cortizo, J.C., Carrero, F. \& al. (2011). Gamificación y Docencia: Lo que la Universidad tiene que aprender de los Videojuegos. Trabajo presentado en VIII Jornadas Internacionales de Innovación Universitaria UEM. Recuperado de http://goo. $\mathrm{gl} / \mathrm{cXqHOp}$

Cuadrado, A. (2011). Utopías y distopías de los medios digitales para la educación. Icono 14, 9 (2), 05-20. doi: 10.7195/ri14.v9i2.31

De-Antonio, A., Villalobos, M. \& Luna, E. (2000). Cuándo y cómo usar la realidad virtual en la enseñanza. Revista de Enseñanza y Tecnología, Enero-Abril, 26-36. 
Dong, X., Yoshida, K. \& Stoffregen, T.A. (2011). Control of a virtual vehicle influences postural activity and motion sickness. Digital Conservancy University of Minessota. Recuperado de http://goo.gl/uPbmXG

E.A.E. (2015). El mercado de videojuegos 2014. Recuperado de http://goo.gl/ msjbvY

Eamshaw, R.A., Gigante, M.A. \& Jones, H. (1993). Virtual Reality Systems. London: Academic Press Ltd.

González, A.L. \& Chávez, G. (2011). La realidad virtual inmersiva en ambientes inteligentes de aprendizaje. Un caso en la educación superior. Icono 14, 9 (2), 05-20. doi: 10.7195/ri14.v9i2.42

Guerrero, B. \& Valero, L. (2013). Efectos secundarios de la realidad virtual inmersiva. International Journal of Psychology and Psychological Therapy, 13 (2), 163-178. Havron, M.D. \& Butler, L.F. (1957). Evaluation of training effectiveness of the 2FH helicopter flight trainer research tool. New York: Naval Training Device Center Project.

Heim, M. (1993). The metaphisics of virtual reality. New York: Oxford University Press. Howart, P.A. \& Hodder, S.G. (2008). Characteristics of habituation to motion in a virtual environment. Displays, 29, 117-123.

Jerónimo, J.A., Andrade, L.C. \& Robles, A. (2011). El diseño educativo en los mundos virtuales. La curva de aprendizaje inmersivo. Icono 14, 9 (2), 05-20. doi: 10.7195/ri14.v9i2.47

Kalawsky, R.S. (1993). The Science of Virtual Reality and Virtual Environments. Reading: Adison-Wesley.

Kenedy, R.S., Lane, N.E., Lilienthal, M.G., Berbaum, K.S. \& Hettinger, L.J. (1992). Profile Analysis of simulator sickness symptoms: Application to virtual environment systems. Presence, 1, 295-301.

Krueger, M. (1983). Artificial Reality. Reading: Addison-Wesley.

Krueger, M. (1985, abril). Videoplace - an artificial reality. Presentado en ACM Conference on Human Factors in Computing Systems.

Lanier, J. (1988). Real Virtuality (entrevista). Whole Earth Review. Recuperado de http://goo.gl/ojKC8f

Lévy, P. (1999). ¿Qué es lo virtual? Barcelona: Paidós.

Matloff, N.S. (1988). Probability modelling and computer simulation an integrated introduction with applications to engineering and computer science. Boston: PWS-Kent. 


\section{ARTÍCULOS DE INVESTIGACIÓN}

Mchaney, R. (1991). Computer Simulation. A Practical Perspective. San Diego: Academic Press.

M.E.C.D. (2014). Cuenta Satélite de la Cultura en España. Avance de resultados 2008-2012. Recuperado de http://goo.gl/Z3GsQo

M.E.C.D. (2015). Encuesta de hábitos y prácticas culturales 2014-2015. Recuperado de http://goo.gl/HIhqei

Otero, A. \& Flores, J. (2011). Realidad virtual: un medio de comunicación de contenidos. Aplicación como herramienta educativa y factores de diseño e implantación en museos y espacios públicos. Icono 14, 9 (2), 05-20. doi: 10.7195/ri14.v9i2.28

Pantelidis, V. (1996). Suggestions on when to use and when not to use virtual reality in education. $V R$ in the schools, 2 (1), p. 18.

Parés, N. \& Parés, R. (2010). Realidad Virtual. Barcelona: FUOC.

Poole, T.G. \& Szymankiewicz, J.C. (1977). Using Simulation to Solve Problems. New York: McGraw-Hill.

Regan, E.C. (1995). An investigation into nausea and other side-effects of headcoupled immersive virtual reality. Virtual Reality, 1, 17-31.

Regan, E.C. \& Price, K.R. (1993). Some side-effects of immersion virtual reality: An investigation into the relationship between distance and ocular related problems. Army Personnel Research Establishment Report, 93-R-023.

Repperger, D.W., Gilkey, R.H., Green, R., Lafleur, T. \& Haas, M.W. (2003). Effects of haptic feedback and turbulence on landing performance using an inmersive cave automatic virtual environment CAVE. Perceptual and Motor Skills, 97, 820-832.

Ripley, B. D. (1987). Stochastic Simulation. New York: John Wiley.

Shannon, R.E. (1975). System Simulation: The Art \& Science. Englewood Cliffs, NJ: Prentice-Hall.

Sharples, S., Cobb, S., Moody, A. \& Wilson, J.R. (2008). Virtual reality induced symptoms and effects: Comparison of head mounted display, desktop and projection display systems. Displays, 29, 58-69.

Sheridan, T.B. (1992). Musings on the Psychophysics of Presence. Presence: Teleoperators and Virtual Environments, 1 (1), 120-126.

Sheridan, T.B. (1996). Futher Musings on the Psychophysics of Presence. Presence: Teleoperators and Virtual Environments, 5 (2), 241-246. doi: 10.1162/ pres.1996.5.2.241 
Slater, M. \& Usoh, M. (1993). Representations Systems, Perceptual Position, and Presence in Immersive Virtual Environments. Presence: Teleoperators and Virtual Environments, 2 (3), 221-233.

Steuer, J. (1992). Defining Virtual Reality: Dimensions Determining Telepresence. Journal of Communication, 42, 73-93.

Sturman, D.J. \& Zeltzer, D. (1994). A survey of glove-based input. IEEE Computer Graphics and Applications, 14 (1), 30-39. doi: 10.1109/38.250916

Sutherland, I. (1968). A Head-Mounted Three-Dimensional Display. En AFIPS Conference Proceedings, 33 (1), 757-764.

U.C.M. (2014). Inserción laboral de titulados Complutense 2014. Recuperado de https://goo.gl/6IZwsT

U.C.M. (2015). Cuestionario de satisfacción. Recuperado de http://goo.gl/649KW6 Ungs, T.J. (1987). Simulator sickness: evidence of long-term effects. En Proceeding of the 31st. Annual Human Factors Society (505-509). Santa Mónica: Human Factors Society.

Vince, J. (1995). Virtual Reality Systems. London: Addison-Wesley.

Walker, J. (1988). Through the looking glass. Recuperado de https://goo.gl/D6ANHa Whicker, M.L. \& Sigelman, L. (1991). Computer simulation applications an introduction. Newbury Park, CA: Sage. 